\title{
RMCProfile: Moving closer to complex modelling
}

\author{
Matt Tucker \\ Spallation Neutron Source, Oak Ridge National Laboratory, Oak Ridge, TN 37831, USA
}

The importance of local structure and disorder in crystalline materials is being recognised more and more as a key property of many functional materials. From negative thermal expansion to solid state amorphisation and the 'nanoscale' problem to improved fuel cell technology, a clear picture of the local atomic structure is essential to understanding these phenomena and solving the associated problems.

Total scattering, an extension of the powder diffraction method, is increasingly being used to study crystalline materials. The unique combination of Bragg and diffuse scattering can be used to determine both the average structure and the short-range fluctuations from this average within a single experiment. To maximise the structural information from such data, three-dimensional atomistic models consistent with all aspects of the data are required.

RMCProfile $^{[1]}$ expands the reverse Monte Carlo (RMC) modelling technique ${ }^{[2]}$ to take explicit account of the Bragg intensity profile from crystalline materials. Analysis of the RMCProfile-generated atomistic models gives more detailed information than is available directly from the data alone.

As the systems being studied become more complex, the information from many experimental techniques and any prior chemical knowledge needs to be combined into one consistent atomic model. The continued development of RMCProfile and its new capabilities are moving us closer to the complex modelling paradigm ${ }^{[3]}$ required to drive the discovery of new functional materials.

[1] see www.rmcprofile.org; M G Tucker, D A Keen, M T Dove, A L Goodwin and Q Hui, J. Phys. Condens. Matter 19, 335218 (2007)

[2] R L McGreevy amd L Pusztai, Mol. Simul. 1, 359 (1988)

[3] S J L Billinge, I Levin, Science, 316, 561 (2007)

Contact: tuckermg@ornl.gov 